\title{
Prediction of attosecond light pulses in the VUV range in a high-order-harmonic-generation regime
}

\author{
Jost Henkel, ${ }^{1,2}$ Tobias Witting, ${ }^{1}$ Davide Fabris, ${ }^{1}$ Manfred Lein, ${ }^{2}$ Peter L. Knight, ${ }^{1}$ \\ John W. G. Tisch, ${ }^{1}$ and Jonathan P. Marangos ${ }^{1}$ \\ ${ }^{1}$ Blackett Laboratory, Imperial College London, Prince Consort Road, South Kensington, London SW7 2AZ, United Kingdom \\ ${ }^{2}$ Institut für Theoretische Physik and Centre for Quantum Engineering and Space-Time Research (QUEST), \\ Leibniz Universität Hannover Appelstraße 2, 30167 Hannover, Germany
}

(Received 11 December 2012; published 15 April 2013)

\begin{abstract}
Attosecond light pulses within the vacuum ultraviolet (VUV) energy range are predicted by solving the time-dependent Schrödinger equation (TDSE) for a model neon atom in short laser pulses of different field polarization states. We compare high-order harmonic generation in linearly polarized laser pulses to the method of polarization gating and find attosecond pulses that approach the Fourier limit of 700 as given by an indium filter, spectrally centered at $15 \mathrm{eV}$. At such low energies, harmonic generation has low sensitivity to ellipticity, which enables the generation of elliptically polarized attosecond pulses. We also show that emission at the atomic transition energies is strongly damped by including intensity averaging.
\end{abstract}

DOI: 10.1103/PhysRevA.87.043818

PACS number(s): 42.65.Ky, 32.80.Rm

\section{INTRODUCTION}

Advances in high-order harmonic generation (HHG) [1,2] over the last decade now allow the creation of previously unrealizable short pulses on the attosecond (as) time scale $[3,4]$. The unique nonlinear response of atoms or molecules allows an up-conversion of the applied infrared (IR) laser field, resulting in the emission of coherent radiation spanning an energy range up to $100 \mathrm{eV}$ and above [5]. Selection of the high-energy part results in the generation of extreme ultraviolet (XUV) attosecond light pulses. To investigate electron dynamics initiated by such XUV pulses in a pumpprobe manner, an intense near-IR field with a stabilized carrier envelope phase (CEP) can be used as a probe. This technique, "attosecond streaking," was successfully used to characterize attosecond pulses [6], for measuring Auger processes [7], and photoemission from solids [8].

The great success of femtosecond (fs) pump-probe techniques in chemistry is based on the efficient selective excitation of a narrow energy band and successive probing with high temporal resolution, which allows the transient characterization and control of atomic motion, even in complicated molecules [9]. Transferring this methodology to the attosecond regime is not straightforward with current XUV pulses, due to their low power, spectrally broad distribution, and the low excitation cross section of molecules under interest. In the case of direct two-photon ionization an application of XUV pump-probe was possible [10]. But to overcome the general limitations in the investigation of electron and hole dynamics and step closer towards attosecond pump-probe experiments, the generation and application of vacuum ultraviolet (VUV, 10-20 eV) pulses with sub-fs temporal duration as a pump pulse is proposed. The collinear generation of both UV and XUV light was demonstrated experimentally [11], although no details of the temporal structure have been reported.

The generation of single as pulses has been achieved using two different approaches. The nonlinear dependence of the HHG on the subcycle intensity of the generating IR field enables the selection of time and energy range by limiting the contributions to a single laser half-cycle. This can be done by compressing the IR field down to waveform-stabilized sub-two-cycle pulses [4]. To overcome stability issues and allow an easier access to as pulses, "polarization gating" approaches using multicycle laser pulses have been demonstrated that rely on the strong ellipticity dependence of HHG [12-14].

In HHG, ionization of the target atom creates an electron wave packet, which is subsequently accelerated back towards the ion by the IR field, and up-converted light is emitted upon recollision. In linearly polarized light ionization during a subcycle time window around the peak of the electric field leads to recollision about one half-cycle later. From classical [15] and quantum-mechanical analysis [16,17] a set of short and long electron trajectories [18] can be obtained, which show the relation between ionization and excursion time, and the recollision energy. The strength of the emitted radiation is modulated by effects at the atomic level, such as the ionization cross section and wave packet spreading during the electron's time in the continuum. HHG also depends on phase-matching conditions as both the drive IR field and the harmonic radiation propagate through the spatially extended target medium [19].

The VUV energy range lies below or around the ionization threshold $I_{p}$ for most atoms, including the most commonly used rare gases helium, neon, and argon. Below threshold harmonics have been investigated both experimentally [20-22] and theoretically [23,24].

In this paper we use the time-dependent Schrödinger equation (TDSE) to calculate the response to short IR fields both from a single atom as well as after intensity averaging. We are interested in the low photon energy (VUV) regime using high-intensity few-cycle drive fields to generate sub-fs pulses with high photon flux compared to available XUV pulses. The requirement of using an intense few-cycle pulse prohibits the use of a perturbation based approach and the focus on the below threshold harmonics is not compatible with the approximations made in the strong-field approximation [16] and derived models such as the quantum orbit model [17]. Although extensions to these models have been proposed, the TDSE is the method that naturally treats the low-energy region correctly. We will, however point out a problem of the singleatom response obtained from the TDSE in the semiclassical approximation: The undamped dipole oscillations lead to 
arbitrarily high emission at the atomic transition energies. We then show that by including intensity averaging this problem disappears.

The strong ellipticity dependence of HHG has long been established. Recombination and thus the observation of HHG in atoms is only possible for small values of the ellipticity [25]. For an elliptically polarized drive field one expects in general HHG contributions polarized along both spatial directions [26]. This has been investigated both in atoms [27,28] and molecules [29-31]. We will show that the time-dependent polarization state of the IR field can be mapped onto an attosecond VUV pulse, generated in an atomic medium.

The numerical methods we use are introduced in Sec. II. In Sec. III we show that the VUV pulses from linearly polarized IR fields approach the Fourier limit given by an indium filter. In Sec. IV we compare the VUV pulses obtained from the polarization-gating method to the results from the previous section and find sub-fs pulses over a wide range of CEP values. There is also a strong correlation between the ellipticity of the generating IR field and the generated VUV pulse. In Sec. V we summarize our conclusions.

\section{METHOD}

We perform TDSE simulations in the single-active electron approximation in a two-dimensional (2D) model for the neon atom for few-cycle pulses with various polarizations. The TDSE reads [atomic units (a.u.) are used in the Method section, i.e., $\left.\hbar=e=m_{e}=1\right]$

$$
i \partial_{t} \psi(x, y, t)=\left[-\nabla^{2} / 2+V(r)+W(x, y, t)\right] \psi(x, y, t),
$$

where $W(x, y, t)$ is the laser-atom interaction in the dipole approximation. The smoothed atomic potential $V(r)$ is chosen to represent the energies of the neon ground state $\left(2 p^{6}\right.$, $-E_{2 p^{6}}=I_{p}=0.792$ a.u. $\left.=21.56 \mathrm{eV}\right)$ and first excited state $\left(2 p^{5} 3 s,-E_{2 p^{5} 3 s}=0.173\right.$ a.u. $\left.=4.71 \mathrm{eV}\right)$ :

$$
V(r)=-1 / \sqrt{r^{2}\left(1+0.62 e^{-0.346 r}\right)+0.038},
$$

with $r^{2}=x^{2}+y^{2}$. The wave function is propagated using the split-operator method [32] on a Cartesian grid, which spans a length of 100 a.u. in each direction with a spatial step size of 0.2 a.u. The time step is 0.007 a.u. Absorbing boundary conditions as in [33] are employed to avoid unphysical reflection or transmission at the grid borders.

The laser-atom interaction is taken in the lengthgauge $W(x, y, t)=\mathbf{E}(t) \cdot \mathbf{r}=\operatorname{Re}\{\tilde{\mathbf{E}}(t)\} \cdot \mathbf{r}$. The initial electric field is

$$
\tilde{\mathbf{E}}_{\mathrm{in}}(t)=E_{0} \cos \left(\omega_{L} t / 8\right)^{2} \exp \left[i\left(\omega_{L} t+\phi\right)\right] \mathbf{e}_{\mathbf{x}},
$$

with the carrier-envelope phase (CEP) $\phi$. Laser parameters are taken as those that can currently be realized in our laboratory [34-36]. The central laser frequency $\omega_{L}$ is 0.0570 a.u., corresponding to a wavelength of $800 \mathrm{~nm}$ (wave period $T_{L}=2 \pi / \omega_{L} \approx 2.6 \mathrm{fs}$ ). The field amplitude $E_{0}$ is chosen as 0.119 a.u. to represent a peak intensity of $I_{0}=5.0 \times 10^{14} \mathrm{~W} / \mathrm{cm}^{2}$. The chosen four-cycle envelope yields a full width at half maximum (FWHM) of $\left(16 / \omega_{L}\right) \arccos \left(2^{-1 / 4}\right)=3.9 \mathrm{fs}$.
For the description of the polarization gating technique we follow the notation of Sansone [37,38]. The polarization state of the incoming linearly polarized IR field $\tilde{\mathbf{E}}_{\mathrm{in}}(t)$ is modified by means of two hypothetical birefringent optical media: First is a quartz plate, oriented at an angle $\alpha$ with respect to the polarization direction of the incoming pulse. Quartz wedges can be used to fine tune the delay $\delta$ introduced between the ordinary and extraordinary pulse components. For a delay corresponding to an odd multiple of $T_{L} / 4$ the quartz acts as a multiple-order quarter-wave plate. The second element is a zero-order quarter-wave plate oriented at an angle $\beta$ with respect to $\tilde{\mathbf{E}}_{\text {in }}$. For the configuration $\alpha=45^{\circ}$ and $\beta=0^{\circ}$, on transiting the second element the field $\tilde{\mathbf{E}}_{\mathrm{g}}(t)$ will comprise two circularly polarized components of opposite helicity with a mutual delay $\delta$. In the case of a short pulse the sum of these components leads to the time varying polarization utilised for gating; i.e., the polarization changes from circular polarization of one helicity to circular polarization of the opposite helicity via a short interval when the field is linearly polarized:

$$
\tilde{\mathbf{E}}_{\mathrm{g}}(t)=\frac{1}{2}\left(\begin{array}{c}
\tilde{E}_{\mathrm{in}, x}(t+\delta / 2)+\tilde{E}_{\mathrm{in}, x}(t-\delta / 2) \\
i\left[\tilde{E}_{\mathrm{in}, x}(t+\delta / 2)-\tilde{E}_{\mathrm{in}, x}(t-\delta / 2)\right]
\end{array}\right) .
$$

The time-dependent ellipticity of a pulse can be calculated from its complex components $\tilde{E}_{x}(t)=\left|\tilde{E}_{x}(t)\right| e^{i \varphi_{x}(t)}$ and $\tilde{E}_{y}(t)=\left|\tilde{E}_{y}(t)\right| e^{i \varphi_{y}(t)}$ as

$$
\epsilon(t)=\tan \left\{\frac{1}{2} \arcsin \left[\frac{2\left|\tilde{E}_{x}\right|\left|\tilde{E}_{y}\right| \sin \left(\varphi_{y}-\varphi_{x}\right)}{\left|\tilde{E}_{x}\right|^{2}+\left|\tilde{E}_{y}\right|^{2}}\right]\right\} .
$$

The HHG signal is calculated from the dipole acceleration [39]

$$
\mathbf{a}(t)=\langle\psi(t)|\nabla V+\mathbf{E}(t)| \psi(t)\rangle,
$$

where the HHG spectrum is then given as

$$
S(\omega)=\left|\int d t \mathbf{a}(t) e^{i \omega t}\right|^{2}=|\tilde{\mathbf{a}}(\omega)|^{2},
$$

where $\tilde{\mathbf{a}}(\omega)$ denotes the Fourier transform of $\mathbf{a}(t)$.

The Gabor transform, a windowed Fourier transform, has been introduced as a way to collect information about the transient emission of each frequency [40,41], which corresponds in the HHG three-step model to the recombination step of electron wave packet and ion. The time-frequency distribution is given as

$$
G(\omega, t)=\left|\int d \tau \mathbf{a}(\tau) e^{i \omega \tau} e^{-(t-\tau)^{2} /\left(2 \sigma^{2}\right)}\right|^{2},
$$

with the window width chosen here as $\sigma=1 /\left(3 \omega_{L}\right)$.

To simulate some effects of the macroscopic propagation of the laser beam through the generating medium $[19,42]$, we follow a procedure called intensity averaging $[43,44]$. As the peak intensity is only reached in the center of the beam profile, many contributions to the total HHG spectrum are made by atoms spatially located in regions with lower intensity. By calculating a coherent summation over intensities $I_{j}$ in an appropriate intensity range we can simulate the dipole phase contributions to the phase-matching effects [45]. The effective 


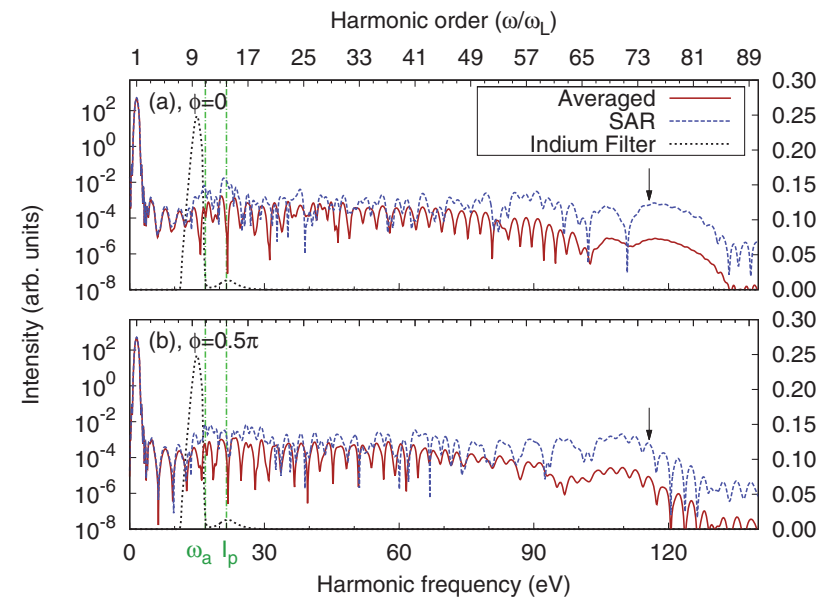

FIG. 1. (Color online) HHG spectra for the neon model generated with a linearly polarized IR field for two different CEPs $\phi=0.0$ (a) and $\phi=0.5 \pi$ (b). Shown are the results from the SAR (blue dashed line) and after intensity averaging (red line). The black dotted line shows the indium filter. The arrow indicates the cutoff for the peak intensity, calculated as $I_{p}+3.2 U_{p}$, with the ponderomotive potential $U_{p}$.

dipole acceleration is then given as

$$
\mathbf{a}_{\mathrm{eff}}(t)=\frac{1}{N} \sum_{i=1}^{N} \mathbf{a}_{I_{i}}(t) .
$$

Calculating the HHG spectrum one finds

$$
\begin{aligned}
S_{\mathrm{eff}}(\omega) & \propto\left|\int d t \sum_{i=1}^{N} \mathbf{a}_{I_{i}}(t) e^{i \omega t}\right|^{2} \\
& =\left|\sum_{i=1}^{N} \int d t \mathbf{a}_{I_{i}}(t) e^{i \omega t}\right|^{2}=\left|\sum_{i=1}^{N} \tilde{\mathbf{a}}_{I_{i}}(\omega)\right|^{2},
\end{aligned}
$$

where it becomes clear that the spectral phase causes interference between contributions from different intensities. It has been shown that this favors the short trajectories [46], which corresponds to an experimental configuration of positioning the gas jet after the laser focus [47].

Filters are used to create attosecond pulses by selecting a suitable energy range. Normally a combination of metal foil and multilayer mirror act as a bandpass filter by offering a suitable transmission range for this task. Their negative group delay dispersion can also compensate $[12,48]$ the positive chirp of the high harmonics from their short trajectories [49]. The group delay dispersion has not been taken into account in the present calculations, as values of the refractive indices are not available for the energy range under consideration. However, the future availability of VUV pulses could in principle make the measurement of the refractive indices feasible [48]. For the VUV range only a few narrow-band metal filters are available. For our calculations we choose an indium foil, which has a maximum in transmission at $15 \mathrm{eV}$ and a bandwidth (FWHM) of $3.7 \mathrm{eV}$ (Fig. 1) [50]. This supports an attosecond pulse with a Fourier-limited duration of about 700 as. We have neglected the transmission of the filter above $100 \mathrm{eV}$, as in a future experiment we aim to optimize emission in the low energy range by phase matching.

\section{LINEAR POLARIZATION}

In this section we will investigate whether the single atom response with the given linearly polarized drive field can generate an attosecond pulse close to the Fourier limit, and how the pulses change by application of the intensity-averaging procedure.

In Fig. 1 we show the calculated HHG spectra for two different CEPs of the generating IR pulse for the single-atom response (SAR) and the intensity-averaged signal, respectively. In the SAR, no clear harmonic peaks are visible, except for the third, fifth, and seventh harmonic, where the system is transparent, as the lowest energy transition only occurs at $E_{2 p^{5} 3 s}-E_{2 p^{6}}=\omega_{a}=16.85 \mathrm{eV}$. The second exception is the cutoff region as there is no interference between short and long trajectories in this region. Instead the interference of contributions from two cycles is visible above the cutoff for a sine-shaped waveform $[\phi \approx \pi / 2 \pm n \pi$, Fig. 1(b)]. In the case of a cosine-shaped IR field $[\phi \approx 0 \pm n \pi$, Fig. 1(a) $]$ the cutoff of the spectrum extends furthest. The smooth continuum at the cutoff has been used to create as pulses in the XUV region $[35,36,51]$.

After intensity averaging we find a number of features upon comparison with the SAR. The low energy harmonics below $\omega_{a}$ show almost no change. As phase-matching conditions only select the radiation that is emitted at the right time in the correct direction, this indicates that these below-absorption harmonics have no contributions with an intensity-dependent phase, but only depend on the phase of the laser field. The protruding structures in the atomic absorption energy range between $\omega_{a}$ and $I_{p}$ disappear and instead harmonic peaks become visible. This will be explained further below. In the plateau region, above the ionization threshold, well-defined peaks become visible. The structure of the cutoff region remains unchanged but the signal is relatively weak, as lower IR intensities make no contributions due to the cutoff scaling. Comparing the peak positions while changing the CEP a variation in energy becomes visible (see also Fig. 2) for HHG above about $40 \mathrm{eV}$ and in particular the cutoff region. These peaks are not integer multiples of $\omega_{L}$ and thus differ from the definition of high harmonics [52]. This analysis shows that the TDSE and the intensity averaging procedure can reproduce established features in HHG.

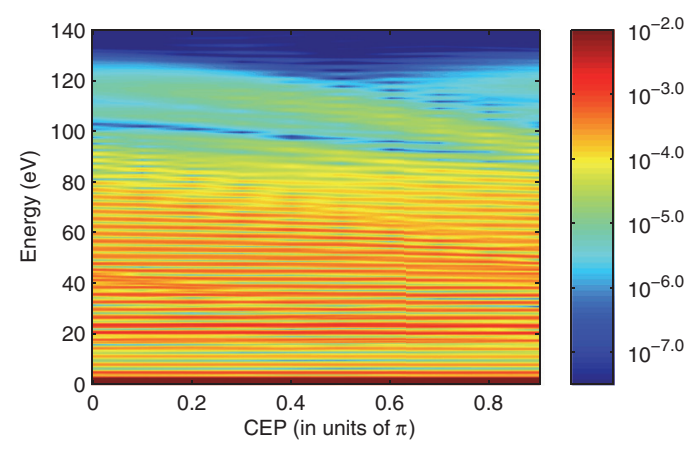

FIG. 2. (Color online) HHG spectra generated from the neon model irradiated with a linearly polarized pulse as a function of the CEP $\phi$ after intensity averaging (logarithmic color scale). The dependence on the CEP for energies above $40 \mathrm{eV}$, in particular in the cutoff, can be clearly seen. 


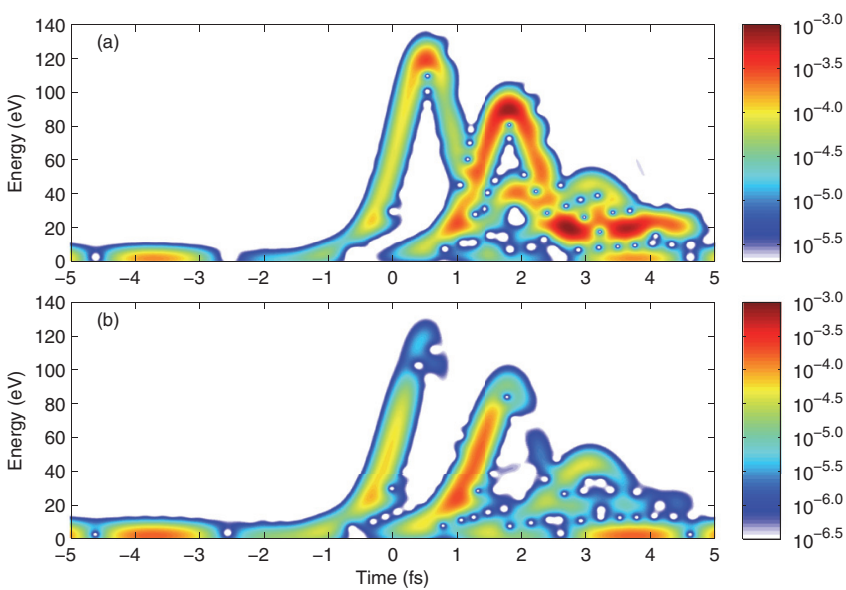

FIG. 3. (Color online) Gabor distributions $G(\omega, t)$ for the linearly polarized IR field with CEP $\phi=0$ (logarithmic color scale). (a) Single-atom response (SAR), (b) after intensity averaging.

We point out a limitation of the TDSE [Eq. (1)]: It does not include spontaneous decay within the semiclassical approximation which treats the electron quantum mechanically, whereas the electromagnetic pulse only enters as a classical field [53]. This means that a superposition of two eigenstates in a system without any external fields seems to radiate forever at the respective transition energy, while all state populations are conserved. In our calculations a Welch window was applied to the dipole acceleration [54] to filter radiation emitted after passage of the IR field. Although the transition lines are not clearly visible in the calculated HHG spectra from the linearly polarized IR field (Fig. 1), their Gabor transform [Fig. 3(a)] shows their contribution around $20 \mathrm{eV}$ after the typical HHG structure. The respective time-frequency graph after intensity averaging [Fig. 3(b)] shows that the intra-atomic transitions as well as the long trajectories are greatly suppressed. This explains the appearance of harmonic peaks below and in the plateau after intensity averaging, as only contributions from the short trajectories interfere.

We now turn to the predicted VUV pulses after applying the indium filter. They show a FWHM temporal duration between a few fs and down to 700 as [Fig. 4(a)]. While a cos-shaped IR field gives short VUV pulses, the rise of a second strong HHG generating cycle, occurring for a sin-shaped field, increases the pulse duration to more than $2 \mathrm{fs}$. This is analogous to the XUV pulses created from the cutoff [51]. The root mean square (RMS), which takes all pulse contributions into account, shows values between 0.9 and 1.3 fs for all CEPs. This means that even though the FHWM for the cos-shaped IR field is small, the pulse shape deviates from the ideal Gaussian shape [Fig. 4(b)]. This indicates contributions from the adjacent half-cycles in the IR field, as can be seen in the Gabor distribution at $15 \mathrm{eV}$ (Fig. 3). Such contributions are absent in the XUV pulse created from the cutoff region (above $100 \mathrm{eV}$ ), shown for comparison in Fig. 4(b). Note the linear intensity scale for the generated pulses in contrast to the logarithmic color scale in Fig. 3.

The VUV pulses obtained from the atomic response exhibit a similar pulse structure shortly after the maximum of the IR field, but they contain strong contributions from
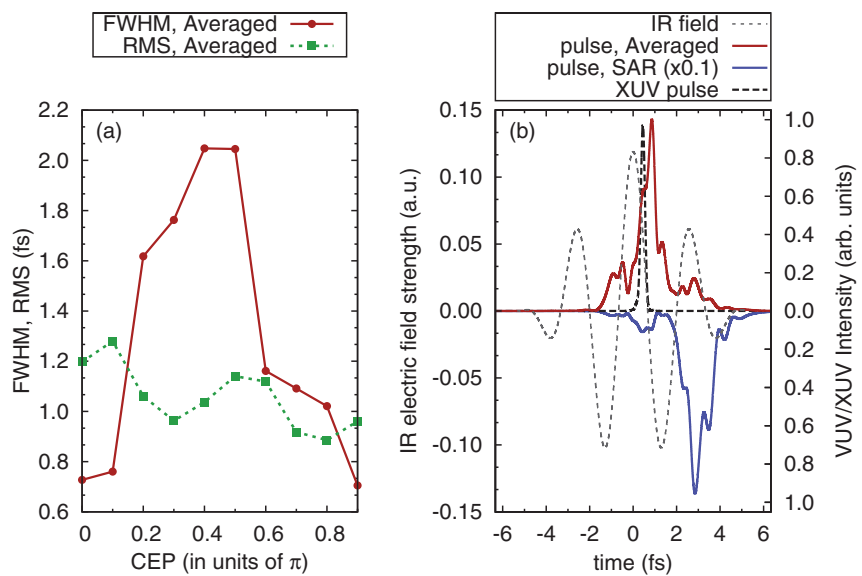

FIG. 4. (Color online) (a) Temporal duration of the predicted VUV pulses (FWHM, red solid line and RMS, green dotted line) from a linearly polarized IR field for the intensity averaged signal. (b) IR field with $\phi=0$ (gray dotted) and the VUV-pulse intensities predicted for a single atom (SAR, blue solid line, downwards and down-scaled for clarity) and the averaging model (red solid line). Also shown for comparison is the XUV pulse obtained from filtering the cutoff region (black dashed line).

the bound states. The temporal width is only limited by the Welch window. Therefore the SAR results cannot be used to make predictions for VUV pulse durations. It is reassuring to see the bound-bound transitions disappear after intensity averaging, while the physically relevant pulse close to midpulse remains almost unchanged. Our calculations suggest that the experimental absence of high-harmonic radiation at the atomic transition energies is at least partially related to poor phase matching of this radiation.

We showed that the FWHM value of the Fourier limit of the indium filter can be reached in our model neon atom over a CEP range of about $0.2 \pi$ (Fig. 4). The pulse shape exhibits small shoulders, as indicated by the RMS value. To make the short pulse length available over a broader CEP range, which would permit a larger experimental CEP fluctuation, while possibly achieving a cleaner pulse shape, we turn towards the polarization gating technique in the next section.

\section{POLARIZATION GATING}

In the following section we will study in detail the HHG process with the polarization gating technique. We investigate whether it can produce cleaner pulse shapes while approaching the Fourier limit for the given energy range of the indium filter for a wider range of CEP. We also examine if the large ellipticity threshold of the high harmonic radiation at low energies allows the creation of elliptically polarized pulses. The ellipticity threshold is the value of ellipticity where the harmonic signal is still $50 \%$ of the maximum signal obtained from linear polarization,

In the polarization gating scheme, high harmonic emission happens mainly in a small time window dictated by the changing ellipticity of the generating IR field. Close to linear polarization one expects a burst of HHG to emerge polarized along the main axis. In principle, very low emission is expected along the minor axis since the field amplitude 
in this direction is low when the time-dependent ellipticity falls below the ellipticity threshold. However, the ellipticity threshold increases for lower harmonic orders [55]. Lower harmonic orders thus see a longer time window and one expects significant contributions polarized along the minor axis at low energies.

In the polarization gating method the driving field consists of two equally strong parts, which are circularly polarized with opposite helicities, and separated by a delay $\delta$. In this section we compare different delay values of $\delta / T_{L}=1 / 4$, $3 / 4$, and $5 / 4$. Here the electric field is rotated such that the main axis always points along $\mathbf{e}_{x}$. An increasing delay leads to a reduction in the peak intensity. The initial intensity $I_{0} /\left(10^{14} \mathrm{~W} / \mathrm{cm}^{2}\right)=5.0$ is reduced to $4.9(4.2,3.0)$ for a delay of $\delta / T_{L}=1 / 4(3 / 4,5 / 4)$. But a longer delay also causes a smaller ellipticity window [56], which leads to a trade-off between a high peak intensity and a short time window.

For the intermediate delay $\delta=3 / 4 T_{L}$, the HHG spectra polarized along the main axis [Fig. 5(a)] resemble the spectra generated with a linearly polarized IR field [Fig. 1(a)]. Well-defined low-order harmonics below $\omega_{a}$ in both SAR and the intensity averaged results can be seen. The pronounced signal in the SAR between $\omega_{a}$ and $I_{p}$, which disappears in the averaged spectrum, is related to the excited states. Well-defined peaks become visible whose energies differ only slightly from odd orders in the range up to $40 \mathrm{eV}$. Similar results are found for short $\delta=1 / 4 T_{L}$ and long delay $\delta=5 / 4 T_{L}$, except for higher and lower signal strength and cutoff, respectively (not shown).

The HHG spectrum polarized along the minor axis paints a different picture [Fig. 5(b)]. As expected from the experimentally measured ellipticity thresholds, one sees a rise in signal strength from the cutoff towards lower energy, reaching a maximum in the atomic absorption region between $\omega_{a}$ and $I_{p}$. The SAR signal above $\omega_{a}$ is equally strong as the SAR signal along the main axis, but is significantly weakened by intensity averaging. This indicates an excitation of states with

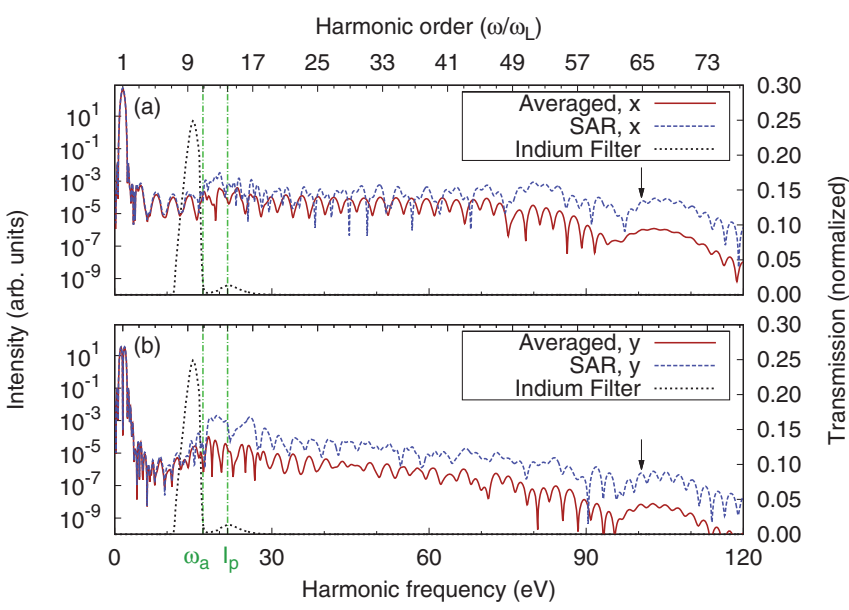

FIG. 5. (Color online) HHG spectra generated with the polarization gating method ( $\delta=3 / 4 T_{L}$ and $\left.\phi=0\right)$ polarized along the main axis (a) and minor axis (b). Shown are the result from the SAR (blue dashed line) and after intensity averaging (red solid line). The black dotted line shows the indium filter. The arrow indicates the cutoff $I_{p}+3.2 U_{p}$ for the peak intensity.
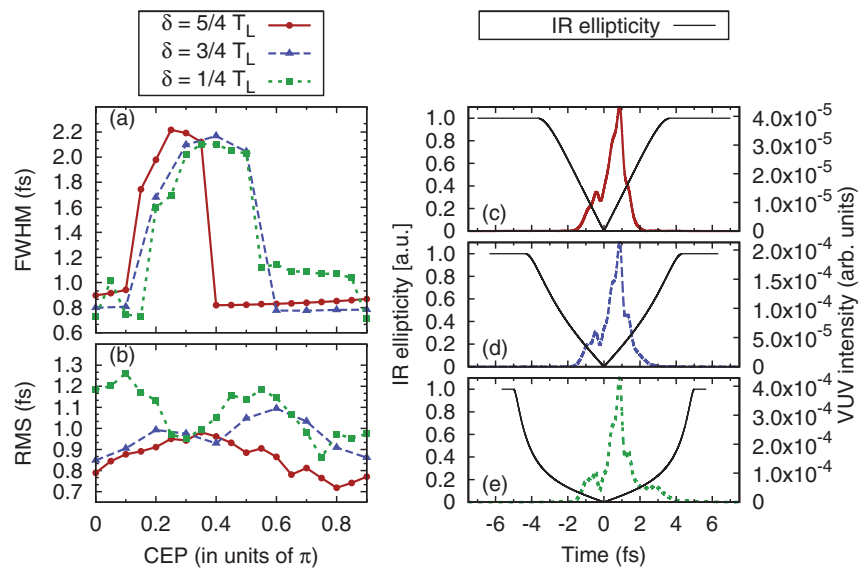

FIG. 6. (Color online) Temporal duration of the VUV pulses [FWHM (a) and RMS (b)] from the polarization gating for the intensity averaged signal. (c)-(e) Absolute value of the ellipticity of the IR field for $\phi=0$ (thin black line) and the intensity profiles of the VUV pulses. Delays are (c) $\delta=5 / 4 T_{L}$ (red solid line), (d) $\delta=3 / 4 T_{L}$ (blue dashed line), and (e) $\delta=1 / 4 T_{L}$ (green dotted line).

a transition dipole moment along the minor axis. Below $\omega_{a}$ the HHG signal drops to a minimum at about $9 \mathrm{eV}$, irrespective of intensity averaging or the delay $\delta$. The appearance of evenorder harmonics after intensity averaging can be explained by the fact that the IR-field component along the minor axis does not change sign between adjacent half-cycles around the change in helicity at $t=0$.

Next, we turn to the pulse durations in the polarization gating scheme. As pointed out above, the $\delta$ parameter offers a compromise between field strength and time window. This becomes apparent in the VUV-pulse duration and intensity. The FWHM and RMS values for the short delay $\delta=1 / 4 T_{L}$ [Figs. 6(a) and 6(b)] reproduce the results obtained for the linearly polarized IR field almost identically [Fig. 4(a)]. The same is true for the corresponding intensity profile for $\phi=0$ [Figs. 6(e) and 4(b)]. The intermediate delay $\delta=3 / 4 T_{L}$ still shows high FWHM values above 1.6 fs for CEPs of $\phi=0.2 \pi$ to $0.5 \pi$, but for the remaining CEP range the FWHM is only about 800 as. This is achieved at the price of half the peak intensity compared to the smaller delay setting, see Fig. 6(d). An even longer delay $\delta=5 / 4 T_{L}$ delivers FWHM values below $1 \mathrm{fs}$ for almost the entire CEP range, except between $\phi=0.15 \pi$ and $0.35 \pi$. The corresponding RMS values below $1 \mathrm{fs}$ indicate clean pulse shapes as can be seen in Fig. 6(c). The short pulses, however, come with a reduction in peak intensity by a factor 5 and 10 compared to the intermediate and short delay, respectively.

In the following we search for imprints of the generating IR-field structure on the VUV-pulse ellipticity. Judging from the weak signal with polarization along the minor axis, only weakly elliptical pulses are expected. However, the HHG component along the minor axis reaches a maximum near $\omega_{a}$. The signal strength at lower energy is weaker, but does not change upon increasing $\delta$. Larger delays nevertheless weaken the main axis signal due to a reduced peak intensity. The selection of the radiation below $\omega_{a}$, as done with the indium filter thus yields $x$ and $y$ components of comparable 

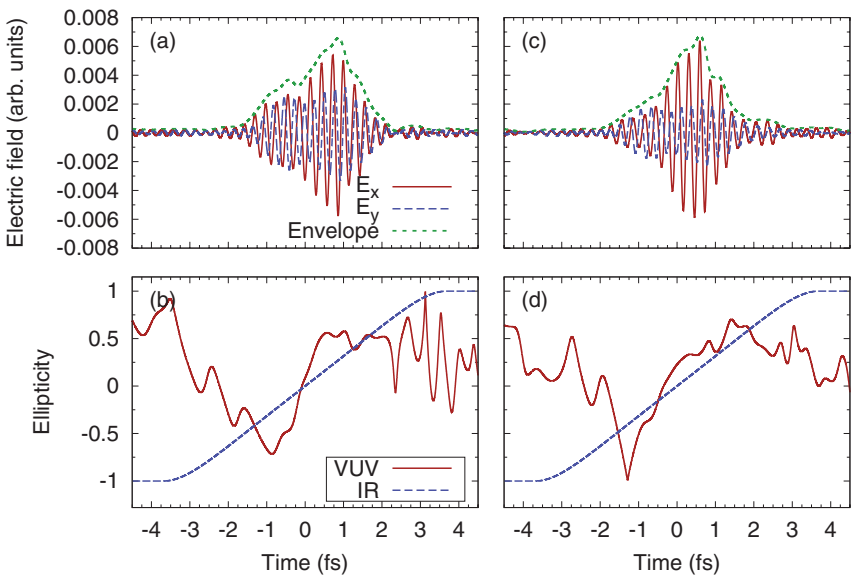

FIG. 7. (Color online) (a) Electric field components of the VUV pulse generated with the polarization gating method $\left(\delta=5 / 4 T_{L}\right.$, $\phi=0)$. Shown are the components polarized along the main axis [ $E_{x}(t)$, red solid line], minor axis [ $E_{y}(t)$, blue dashed line], and their envelope $\left[\sqrt{\left|E_{x}(t)\right|^{2}+\left|E_{y}(t)\right|^{2}}\right.$, green dotted line $]$. (b) Ellipticities of the VUV pulse (red solid line) and the IR field (blue dashed line) calculated from Eq. (5). (c) and (d) Same as (a) and (b) but for $\phi=0.8 \pi$.

strength in the VUV pulse for large delays [Fig. 7(a) and 7(c)]. Analyzing the phase difference between the two components, which defines the helicity of the VUV pulse, one finds that in the vicinity of the ellipticity window the VUV-pulse helicity follows that of the IR field [Figs. 7(b) and 7(d)]. It turns from a left-turning rotation at negative times through linear polarization to a right-turning rotation at positive times. The exact timing for this helicity change varies with the CEP, while the overall structure is preserved, even for the smaller delay values, where the ellipticity is smaller (not shown). Comparing the ellipticities for two different CEPs, $\phi=0$ and $\phi=0.8 \pi$
[Figs. 7(b) and 7(d)], we find the point of linear polarization in the VUV pulse shifted from about -100 to -350 as. The possibility to shift the turning point in time by changing the CEP of the driving pulse, while keeping the pulse length stable, is another step towards pulse shaping in attosecond science as already routinely used in femtosecond science [57,58].

\section{CONCLUSIONS}

We have reported 2D TDSE calculations for a model neon atom driven by few-cycle laser pulses. The calculations predict sub-fs VUV pulses with central energy $15 \mathrm{eV}$ after application of an indium filter, both for a linearly polarized generating IR field and for a polarization gating scheme. Strong emission along the minor axis in short ellipticity windows allows the mapping of ellipticity changes from the IR field onto the VUV pulses. This can lead to advanced pulse-shaping tools in attosecond science. Sub-fs VUV pulses will allow the selective excitation of electronic transitions in molecules which can then be probed by available XUV pulses. This is a important step towards true attosecond pump-probe experiments and will thus offer insight in subatomic processes that have eluded observation due to their short lifetimes. Besides the experimental realization of the VUV pulses, their characterisation also provides a new challenge as existing methods cannot be applied easily.

\section{ACKNOWLEDGMENTS}

This research was supported in part by EPSRC programme grant EP/I032517/1. J.H. acknowledges funding from the EU Marie Curie Initial Training Network FASTQUAST. We acknowledge financial support from the Deutsche Forschungsgemeinschaft. We acknowledge valuable discussions with A. Zaïr and F. Frank.
[1] M. F. Kling and M. J. Vrakking, Annu. Rev. Phys. Chem. 59, 463 (2008).

[2] F. Krausz and M. Ivanov, Rev. Mod. Phys. 81, 163 (2009).

[3] K. Zhao, Q. Zhang, M. Chini, Y. Wu, X. Wang, and Z. Chang, Opt. Lett. 37, 3891 (2012).

[4] E. Goulielmakis, M. Schultze, M. Hofstetter, V. S. Yakovlev, J. Gagnon, M. Uiberacker, A. L. Aquila, E. M. Gullikson, D. T. Attwood, R. Kienberger, F. Krausz, and U. Kleineberg, Science 320, 1614 (2008).

[5] L. E. Chipperfield, J. S. Robinson, J. W. G. Tisch, and J. P. Marangos, Phys. Rev. Lett. 102, 063003 (2009).

[6] Y. Mairesse and F. Quéré, Phys. Rev. A 71, 011401 (2005).

[7] M. Drescher, M. Hentschel, R. Kienberger, M. Uiberacker, V. Yakovlev, A. Scrinzi, T. Westerwalbesloh, U. Kleineberg, U. Heinzmann, and F. Krausz, Nature (London) 419, 803 (2002).

[8] A. L. Cavalieri, N. Müller, T. Uphues, V. S. Yakovlev, A. Baltuka, B. Horvath, B. Schmidt, L. Blümel, R. Holzwarth, S. Hendel, M. Drescher, U. Kleineberg, P. M. Echenique, R. Kienberger, F. Krausz, and U. Heinzmann, Nature (London) 449, 1029 (2007).

[9] A. H. Zewail, J. Phys. Chem. A 104, 5660 (2000).
[10] P. Tzallas, E. Skantzakis, L. a. A. Nikolopoulos, G. D. Tsakiris, and D. Charalambidis, Nat. Phys. 7, 781 (2011).

[11] E. M. Bothschafter, A. Schiffrin, V. S. Yakovlev, A. M. Azzeer, F. Krausz, R. Ernstorfer, and R. Kienberger, Opt. Express 18, 9173 (2010).

[12] G. Sansone, E. Benedetti, F. Calegari, C. Vozzi, L. Avaldi, R. Flammini, L. Poletto, P. Villoresi, C. Altucci, R. Velotta, S. Stagira, S. De Silvestri, and M. Nisoli, Science 314, 443 (2006).

[13] Z. Chang, Phys. Rev. A 76, 051403 (2007).

[14] C. Altucci, J. Tisch, and R. Velotta, J. Mod. Opt. 58, 1585 (2011).

[15] P. B. Corkum, Phys. Rev. Lett. 71, 1994 (1993).

[16] M. Lewenstein, P. Balcou, M. Y. Ivanov, A. L'Huillier, and P. B. Corkum, Phys. Rev. A 49, 2117 (1994).

[17] P. Salieres, B. Carré, L. L. Déroff, F. Grasbon, G. G. Paulus, H. Walther, R. Kopold, W. Becker, D. B. Milosević, A. Sanpera, and M. Lewenstein, Science 292, 902 (2001).

[18] L. Brugnera, D. J. Hoffmann, T. Siegel, F. Frank, A. Zaïr, J. W. G. Tisch, and J. P. Marangos, Phys. Rev. Lett. 107, 153902 (2011). 
[19] M. B. Gaarde, J. L. Tate, and K. J. Schafer, J. Phys. B 41, 132001 (2008).

[20] D. C. Yost, T. R. Schibli, J. Ye, J. L. Tate, J. Hostetter, M. B. Gaarde, and K. J. Schafer, Nat. Phys. 5, 815 (2009).

[21] E. P. Power, A. M. March, F. Catoire, E. Sistrunk, K. Krushelnick, P. Agostini, and L. F. DiMauro, Nat. Photon. 4, 352 (2010).

[22] H. Soifer, P. Botheron, D. Shafir, A. Diner, O. Raz, B. D. Bruner, Y. Mairesse, B. Pons, and N. Dudovich, Phys. Rev. Lett. 105, 143904 (2010).

[23] J. A. Hostetter, J. L. Tate, K. J. Schafer, and M. B. Gaarde, Phys. Rev. A 82, 023401 (2010).

[24] J.-C. Liu, M. C. Kohler, C. H. Keitel, and K. Z. Hatsagortsyan, Phys. Rev. A 84, 063817 (2011).

[25] P. Antoine, A. L'Huillier, M. Lewenstein, P. Salières, and B. Carré, Phys. Rev. A 53, 1725 (1996).

[26] W. Becker, A. Lohr, M. Kleber, and M. Lewenstein, Phys. Rev. A 56, 645 (1997).

[27] F. A. Weihe and P. H. Bucksbaum, J. Opt. Soc. Am. B 13, 157 (1996).

[28] P. Antoine, B. Carré, A. L’Huillier, and M. Lewenstein, Phys. Rev. A 55, 1314 (1997).

[29] J. Levesque, Y. Mairesse, N. Dudovich, H. Pépin, J.-C. Kieffer, P. B. Corkum, and D. M. Villeneuve, Phys. Rev. Lett. 99, 243001 (2007).

[30] X. Zhou, R. Lock, N. Wagner, W. Li, H. C. Kapteyn, and M. M. Murnane, Phys. Rev. Lett. 102, 073902 (2009).

[31] K.-J. Yuan and A. D. Bandrauk, J. Phys. B 45, 074001 (2012).

[32] M. Feit, J. Fleck, Jr., and A. Steiger, J. Comput. Phys. 47, 412 (1982).

[33] J. Henkel, M. Lein, and V. Engel, Phys. Rev. A 83, 051401 (2011).

[34] T. Witting, F. Frank, C. A. Arrell, W. A. Okell, J. P. Marangos, and J. W. G. Tisch, Opt. Lett. 36, 1680 (2011).

[35] T. Witting, F. Frank, W. A. Okell, C. A. Arrell, J. P. Marangos, and J. W. G. Tisch, J. Phys. B 45, 074014 (2012).

[36] F. Frank, C. Arrell, T. Witting, W. A. Okell, J. McKenna, J. S. Robinson, C. A. Haworth, D. Austin, H. Teng, I. A. Walmsley, J. P. Marangos, and J. W. G. Tisch, Rev. Sci. Instrum. 83, 071101 (2012).

[37] G. Sansone, Phys. Rev. A 79, 053410 (2009).

[38] G. Sansone, E. Benedetti, J. P. Caumes, S. Stagira, C. Vozzi, M. Nisoli, L. Poletto, P. Villoresi, V. Strelkov, I. Sola, L. B. Elouga, A. Zaïr, E. Mével, and E. Constant, Phys. Rev. A 80, 063837 (2009).
[39] K. Burnett, V. C. Reed, J. Cooper, and P. L. Knight, Phys. Rev. A 45, 3347 (1992).

[40] D. Gabor, J. Inst. Electr. Eng. 93, 429 (1946).

[41] C. C. Chirila, I. Dreissigacker, E. V. van der Zwan, and M. Lein, Phys. Rev. A 81, 033412 (2010).

[42] M. B. Gaarde, P. Antoine, A. L'Huillier, K. J. Schafer, and K. C. Kulander, Phys. Rev. A 57, 4553 (1998).

[43] T. Morishita, A.-T. Le, Z. Chen, and C. D. Lin, Phys. Rev. Lett. 100, 013903 (2008).

[44] A.-T. Le, T. Morishita, and C. D. Lin, Phys. Rev. A 78, 023814 (2008).

[45] C. Jin, A.-T. Le, and C. D. Lin, Phys. Rev. A 79, 053413 (2009).

[46] M. Tudorovskaya and M. Lein, Phys. Rev. A 84, 013430 (2011).

[47] P. Antoine, A. L'Huillier, and M. Lewenstein, Phys. Rev. Lett. 77, 1234 (1996).

[48] R. López-Martens, K. Varjú, P. Johnsson, J. Mauritsson, Y. Mairesse, P. Salières, M. B. Gaarde, K. J. Schafer, A. Persson, S. Svanberg, C.-G. Wahlström, and A. L'Huillier, Phys. Rev. Lett. 94, 033001 (2005).

[49] Y. Mairesse, A. de Bohan, L. J. Frasinski, H. Merdji, L. C. Dinu, P. Monchicourt, P. Breger, M. Kovacev, R. Täeb, B. Carré, H. G. Muller, P. Agostini, and P. Saliéres, Science 302, 1540 (2003).

[50] B. Henke, E. Gullikson, and J. Davis, At. Data Nucl. Data Tables 54, 181 (1993).

[51] E. Goulielmakis, M. Uiberacker, R. Kienberger, A. Baltuska, V. Yakovlev, A. Scrinzi, T. Westerwalbesloh, U. Kleineberg, U. Heinzmann, M. Drescher, and F. Krausz, Science 305, 1267 (2004).

[52] A. Baltuska, T. Udem, M. Uiberacker, M. Hentschel, E. Goulielmakis, C. Gohle, R. Holzwarth, V. S. Yakovlev, A. Scrinzi, T. W. Hänsch, and F. Krausz, Nature (London) 421, 611 (2003).

[53] K. J. Schafer, Strong Field Laser Physics (Springer, Berlin, 2009), p. 111.

[54] M. Lein, Phys. Rev. A 72, 053816 (2005).

[55] I. J. Sola, E. Mével, L. Elouga, E. Constant, V. Strelkov, L. Poletto, P. Villoresi, E. Benedetti, J.-P. Caumes, S. Stagira, C. Vozzi, G. Sansone, and M. Nisoli, Nat. Phys. 2, 319 (2006).

[56] V. Strelkov, A. Zaï, O. Tcherbakoff, R. López-Martens, E. Cormier, E. Mével, and E. Constant, J. Phys. B 38, L161 (2005).

[57] T. Brixner and G. Gerber, Opt. Lett. 26, 557 (2001).

[58] J. Köhler, M. Wollenhaupt, T. Bayer, C. Sarpe, and T. Baumert, Opt. Express 19, 11638 (2011). 